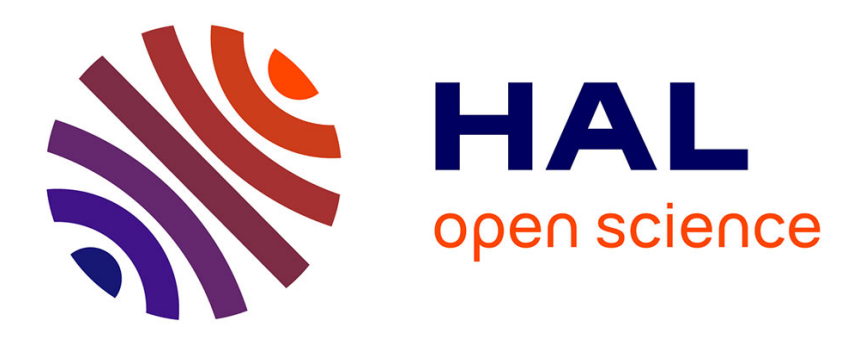

\title{
Metric-independent analysis of the stress-energy tensor Reuven Segev
}

\section{To cite this version:}

Reuven Segev. Metric-independent analysis of the stress-energy tensor. Journal of Mathematical Physics, 2002, 43 (6), pp.3220-3231. hal-00957657

\section{HAL Id: hal-00957657 https://hal.science/hal-00957657}

Submitted on 10 Mar 2014

HAL is a multi-disciplinary open access archive for the deposit and dissemination of scientific research documents, whether they are published or not. The documents may come from teaching and research institutions in France or abroad, or from public or private research centers.
L'archive ouverte pluridisciplinaire HAL, est destinée au dépôt et à la diffusion de documents scientifiques de niveau recherche, publiés ou non, émanant des établissements d'enseignement et de recherche français ou étrangers, des laboratoires publics ou privés. 


\title{
METRIC-INDEPENDENT ANALYSIS OF THE STRESS-ENERGY TENSOR
}

\author{
REUVEN SEGEV
}

\begin{abstract}
The stress-energy tensor of field theory is defined and analyzed in a geometric setting where a metric is not available. The stress is a linear mapping that transforms the 3-form representing the flux of any given property, e.g., charge-current density, to the 3-form representing the flux of energy. The example of the electromagnetic stress-energy tensor is given with the additional structure of a volume element.
\end{abstract}

Keywords. Stress-energy tensor, field theory, differential forms, conservation laws, flux.

\section{INTRODUCTION}

The introduction of the stress-energy tensor in field theory and the related analysis of conservation laws utilize the metric properties of spacetime afforded by relativity theory (see for example $[1,2,3,4,5]$ ). Since one cannot assume that the metric tensor is known in advance, it would be preferable, at least from the theoretical point of view, to have a formulation of the theory that does not rely on the metric structure. Such a presentation of the stress-energy tensor and conservation laws is the subject of this paper.

For the electromagnetic field as a concrete example, the construction may be described simply as follows: the value of stress-energy tensor at any event is a linear mapping that transforms the charge-current density 3-form to the corresponding energy flux 3-form.

The basic geometric setting is that of an $m$-dimensional spacetime manifold $\mathcal{U}$. Spacetime is assumed to be orientable and a specific orientation is assumed to be chosen. In particular, we do not use a metric or a connection in the analysis. The first part of the paper, containing Sections 2 and 3 , is concerned with the fibration of spacetime induced by a conservation law for an extensive property $p$, for example, the electric charge. Assuming that the flux of the property $p$ out of any region $\mathcal{R}$ in spacetime is given as an integral of a flux density $(m-1)$-form $\tau_{\mathcal{R}}$, and using a generalization of the traditional Cauchy assumptions regarding the dependence of the form $\tau_{\mathcal{R}}$ on $\mathcal{R}$, there is a unique flux $(m-1)$-form $J$ (see [6]), the analog of the 
charge-current density 3-form in electromagnetism, such that for any region $\mathcal{R}, \tau_{\mathcal{R}}=\iota^{*}(J)$, where $\iota^{*}$ is the restriction of forms defined on spacetime to the boundary of $\mathcal{R}$. The flux density form induces a 1-dimensional subbundle of the tangent bundle $T \mathcal{U}$ whose integral manifolds are the worldlines associated with the property. Thus, even in this general setting, the conservation of the property induces enough structure so the analogs of particles and velocities-worldlines and flux $(m-1)$-forms-may be defined. If a volume element $\theta$ is given on $\mathcal{U}$, the flux form induces a vector field $v$, the analog of the 4-velocity, by the condition $J=v\lrcorner \theta$.

The next part of the paper, consisting of Sections 4, 5 and 6, presents stress theory on manifolds (see also previous works $[7,8]$ ). Consider a vector bundle $W \rightarrow \mathcal{U}$, whose elements are interpreted as values of generalized velocities. For a region $\mathcal{R}$ in spacetime, Section 4 is concerned, with a linear functional on sections $w$ of $W$ that contains a "volume" term and a boundary term. The boundary term for a region $\mathcal{R} \subset \mathcal{U}$ is given as $\mathbf{t}_{\mathcal{R}}(w)$ where $\mathbf{t}_{\mathcal{R}}$ is a section of the bundle of linear mappings $L\left(W, \bigwedge^{m-1}\left(T^{*} \partial \mathcal{R}\right)\right)$. Again, with the Cauchy postulates for the dependence of $\mathbf{t}_{\mathcal{R}}$ on $\mathcal{R}$, there is a unique section $\sigma$ of $L\left(W, \bigwedge^{m-1}\left(T^{*} \mathcal{U}\right)\right)$, the Cauchy stress, that induces by restriction of forms the vector valued forms $\mathbf{t}_{\mathcal{R}}$ for the various regions.

Section 5 considers a linear functional on sections of $W$ that may be represented as follows. Let $J^{1}(W)$ be the jet bundle associated with $W$. Then, there is a section of $L\left(J^{1}(W), \bigwedge^{m}\left(T^{*} \mathcal{U}\right)\right)$, the variational stress density, such that the value of the functional for a section $w$ is $\int_{\mathcal{R}} S\left(j^{1}(w)\right)$, where $j^{1}(w)$ is the first jet of the section $w$. The divergences of variational stress densities are defined and the relation between Cauchy stresses and variational stresses is presented in Section 6.

The values of the functionals described above are interpreted in Section 7 as the energy variation associated with the motion of the property $p$ as represented by the flux form $J$. Accordingly, $\bigwedge^{m-1}\left(T^{*} \mathcal{U}\right)$ is used for the vector bundle $W$ over spacetime. In this case, the Cauchy stress is a section of $L\left(\bigwedge^{m-1}\left(T^{*} \mathcal{U}\right), \bigwedge^{m-1}\left(T^{*} \mathcal{U}\right)\right)$ - the stress-energy tensor. It is shown in Section 8 that stress-energy tensors can be represented naturally by sections of the bundle of linear mappings $L(T \mathcal{U}, T \mathcal{U})$.

Finally, Section 9 presents the example of the stress-energy tensor for electromagnetism. No particular relation is used for the constitutive relation between the Maxwell and Faraday 2-forms and the only additional geometric structure used is that of a volume element. Mathematically, this enables us to obtain the 4-velocity vector field from the flux form. The expression for the Lorentz force we obtain is analogous to that of [2, p. 91] where a metric is used.

In [9], Gotay \& Marsden present a derivation of a metric independent stress-energy tensor using a different approach. In comparison with the present paper, the authors assume additional structure of a Lagrangian 
and a gauge group. Accordingly, the results they obtain are more comprehensive. The stress object derived in [9] is a $(1,1)$-tensor density, i.e., a section of $L\left(L(T \mathcal{U}, T \mathcal{U}), \bigwedge^{m}\left(T^{*} \mathcal{U}\right)\right)$ that may be identified with a section of $L\left(T \mathcal{U}, \bigwedge^{m-1}\left(T^{*} \mathcal{U}\right)\right)$. Here, allowing a slight generalization where a stress is an element of $L\left(L(W, T \mathcal{U}), \bigwedge^{m}\left(T^{*} \mathcal{U}\right)\right)$ for some vector bundle $W$, and then putting $W=\bigwedge^{m-1}\left(T^{*} \mathcal{U}\right)$ (see Section 7 for the motivation), we arrive at a stress object that is a $(1,1)$ tensor, i.e., a section of $L(T \mathcal{U}, T \mathcal{U})$.

\section{Scalar Valued Extensive Properties on Spacetime}

We consider the conservation of an extensive property $p$ in spacetime $\mathcal{U}$. It is assumed that $\mathcal{U}$ is an $m$-dimensional orientable manifold with a definite orientation chosen. An $m$-dimensional submanifold with boundary $\mathcal{R}$ of $\mathcal{U}$ will be referred to as a control region.

Specifically, it is assumed that for each control region $\mathcal{R}$ there is an $(m-1)$ form $\tau_{\mathcal{R}}$ on $\partial \mathcal{R}$, the flux density. The integral $\int_{\partial \mathcal{R}} \tau_{\mathcal{R}}$ is interpreted as the flux of the property out of the control region in spacetime relative to the positive orientation induced on $\partial \mathcal{R}$ by the orientation on $\mathcal{U}$ and the outwards pointing vectors. In case a frame is given, the flux density through a spacelike slice is interpreted as the density of the property $p$ in space and the flux through a hyperplane containing the $\partial / \partial t$ tangent vector is interpreted as the classical flux density of $p$ into the corresponding slice consisting of simultaneous events.

Regarding $\tau_{\mathcal{R}}$ as the value of a set function defined on the collection of control regions, Cauchy's postulates of continuum mechanics can be generalized to differentiable manifolds as follows (see [6], [8]).

GC1 There is a volume element $\theta$ on $\mathcal{U}$ such that

$$
\left|\int_{\partial \mathcal{R}} \tau_{\mathcal{R}}\right| \leq \int_{\mathcal{R}} \theta
$$

GC2 Consider the Grassmann bundle of hyperplanes $\pi_{G}: G_{m-1}(T \mathcal{U}) \rightarrow$ $\mathcal{U}$ whose fiber $G_{m-1}\left(T_{x} \mathcal{U}\right)$ at any event $x \in \mathcal{U}$ is the Grassmann manifold of hyperplanes, i.e., $(m-1)$-dimensional subspaces of the tangent space $T_{x} \mathcal{U}$. Let $\bigwedge^{m-1}\left(G_{m-1}(T \mathcal{U})\right)^{*} \rightarrow G_{m-1}(T \mathcal{U})$ be the vector bundle over $G_{m-1}(T \mathcal{U})$ whose fiber over a hyperplane $H$ is the vector space of $(m-1)$-forms on $H$. Then, the dependence of $\tau_{\mathcal{R}}$ on $\mathcal{R}$ is via a smooth section

$$
\tau: G_{m-1}(T \mathcal{U}) \rightarrow \bigwedge^{m-1}\left(G_{m-1}(T \mathcal{U})\right)^{*},
$$

such that $\tau_{\mathcal{R}}=\tau\left(T_{x} \partial \mathcal{R}\right)$.

Cauchy's theorem, generalized in [6], [8] to manifolds, states that there is a unique $(m-1)$-form $J$ on $\mathcal{U}$ such that for any control region $\mathcal{R}$,

$$
\tau_{\mathcal{R}}=\tau\left(T_{x} \partial \mathcal{R}\right)=\iota^{*}(J)
$$


Here, $\iota: \partial \mathcal{R} \rightarrow \mathcal{U}$ is the natural inclusion and $\iota^{*}$ is the pull-back of forms it induces. We will refer to $J$ as the flux form associated with the property $p$.

Usually, it is assumed that there is a source density term $s$ for the property, an $m$-form on $\mathcal{U}$, so that the conservation equation of the property is

$$
\int_{\partial \mathcal{R}} \tau_{\mathcal{R}}=\int_{\mathcal{R}} s .
$$

In this case, Stokes' theorem implies that the conservation equation may be written in a differential form as $d J=s$. (Again, if a frame is given on spacetime, then the time component of $J$ is the density in space of the property $p$ and the term in $d J$ containing it is the time derivative of that density. The space-like components of $J$ describe the 3-dimensional flux and the terms in $d J$ involving the space-like components make its $(m-1)$-dimensional divergence.) In a particular frame, for every time $t$, the classical conservation law has the integral form

$$
\int_{\mathcal{R}} \beta_{\mathcal{R}}+\int_{\partial \mathcal{R}} \tau_{\mathcal{R}}=\int_{\mathcal{R}} s,
$$

where here $\mathcal{R}$ is interpreted as a region in space (a slice of spacetime) and $\beta_{\mathcal{R}}$ is the rate of change of the density of the property-a 3-form. In order that the previous Cauchy assumptions apply, it is usually assumed that $\beta_{\mathcal{R}}$ is actually independent of $\mathcal{R}$.

Remark 2.1. Assume the manifold $\mathcal{U}$ is given a particular volume element $\theta$. Then, there is a vector bundle isomorphism

$$
i_{\theta}: \bigwedge^{m-1}\left(T_{x}^{*} \mathcal{U}\right) \rightarrow T_{x} \mathcal{U}
$$

such that $\left.\left(i_{\theta} \circ J\right)\right\lrcorner \theta=J$, where $\lrcorner$ denotes the contraction (interior product) of forms by vectors. If $\theta$ is represented locally by

$$
r\left(x^{i}\right) d x^{1} \wedge \ldots \wedge d x^{m}
$$

then, $v=i_{\theta} \circ J$, which we will also write as $i_{\theta}(J)$, is represented by

$$
v^{i}=\frac{(-1)^{i+1} J_{i}}{r}
$$

If $J$ is a flux form of an extensive property $p$ and a volume element is given, we will refer to $v=i_{\theta}(J)$ as the kinematic flux associated with $p$. The kinematic flux is the analog of the 4 -velocity field. If $\mathcal{L}$ denotes the Lie derivative, then the differential conservation equation can now be written in the form $\mathcal{L}_{v} \theta=s$. 


\section{Worldlines AND GENERALIZED BODY POINTS}

A flux form $J$ induces a 1-dimensional distribution over the open submanifold of $\mathcal{U}$ where it does not vanish. Let $E(J)$ be the minimal enveloping subbundle associated with $J$, i.e., the minimal subbundle $Z$ of $T^{*} \mathcal{U}$ such that $J(x) \in \bigwedge^{m-1} Z_{x}$. We will refer to the annihilator $E(J)^{\perp} \subset T \mathcal{U}$ of the minimal enveloping subbundle as the flux bundle. That is,

$$
E(J)_{x}^{\perp}=\left\{v \in T_{x} \mathcal{U} ; \phi(v)=0 \text {, for all } \phi \in E(J)_{x}\right\} .
$$

The flux bundle is 1-dimensional and a tangent vector $v$ is in the flux bundle if and only if $v\lrcorner J=0$. The flux bundle is also the one dimensional bundle obtained by the relation $v=i_{\theta}(J)$ when the flux form $J$ is kept fixed and the volume element $\theta$ is allowed to vary. Being 1-dimensional, the flux bundle is integrable, and its 1-dimensional integral manifolds will be referred to as (local) worldlines. Consider the equivalence relation $x \sim x^{\prime}$ if $x$ and $x^{\prime}$ are on the same worldline. We will refer to the collection of worldlines $\mathcal{B}=\mathcal{U} / \sim$ as the material universe.

The worldlines form a foliation of $\mathcal{U}$ (See [10] for a detailed treatment). In case the foliation is regular, so $\mathcal{B}$ is an $(m-1)$-dimensional submanifold of $\mathcal{U}$ and the natural projection $\mathcal{U} \rightarrow \mathcal{B}=\mathcal{U} / \sim$ is a submersion, an element of $\mathcal{B}$ is a material point and a compact $(m-1)$-dimensional submanifold with boundary of $\mathcal{B}$ is a material body. A necessary and sufficient condition for the foliation to be regular is the existence of local slices, i.e., at every event $x$ there exists a local $(m-1)$-dimensional submanifold $P$ of $\mathcal{U}$ such that $P$ intersects every worldline at one point at most and $T_{x} \mathcal{U}=T_{x} P \times T_{x} Y$, where $Y$ is the worldline through $x$.

Thus, in case the foliation by worldlines is regular, the construction we described generates a material structure in space even though the velocity field is not defined uniquely. In addition, the flux form $J$ is an object that generalizes the velocity field even if a volume element is not given and even if the foliation it generates is not regular.

Clearly, foliated charts and slices generate frames that assign to events unique material points and "time" coordinates. If a volume element is given, the kinematic flux induces a unique time coordinate in the neighborhood of every event (independently of a chart). Thus, a flux form and a volume element induce together a local frame.

\section{CAuChy's STRESS THEORY FOR MANifOldS}

Let $\pi: W \rightarrow \mathcal{U}$ be a vector bundle over the $m$-dimensional orientable manifold $\mathcal{U}$. The vector bundle is interpreted as the bundle of generalized velocities over $\mathcal{U}$. In classical continuum mechanics, if $\mathcal{U}$ is interpreted as the physical space (a slice of spacetime), then in many cases $W$ is the tangent bundle $T \mathcal{U}$. If $\mathcal{U}$ is interpreted as the material body, then $W$ is usually the pull-back of the tangent bundle of the space manifold under the configuration mapping that embeds the material universe in space. This is the 
interpretation used in previous works (e.g., [7],[8]). In either case, a section of the bundle $\pi$ is interpreted as a generalized velocity field from either the Eulerian or the Lagrangean points of view.

Cauchy's stress theory for manifolds, presented in [8], considers for each compact $m$-dimensional submanifold with boundary $\mathcal{R}$ of $\mathcal{U}$ a linear functional of the generalized velocity fields containing a volume term and a boundary term of the form

$$
F_{\mathcal{R}}(w)=\int_{\mathcal{R}} \mathbf{b}_{\mathcal{R}}(w)+\int_{\partial \mathcal{R}} \mathbf{t}_{\mathcal{R}}(w) .
$$

Here, $w$ is a section of $W, \mathbf{b}_{\mathcal{R}}$, the body force, is a section of $L\left(W, \bigwedge^{m}\left(T^{*} \mathcal{R}\right)\right)$ and $\mathbf{t}_{\mathcal{R}}$ the boundary force is a section of $L\left(W, \bigwedge^{m-1}\left(T^{*} \partial \mathcal{R}\right)\right)$ so the integrals make sense. The functional $F_{\mathcal{R}}$ is interpreted as the force, or power, functional and the value $F_{\mathcal{R}}(w)$ is classically interpreted as the power of the force for the generalized velocity field $w$.

We note that body forces and surface forces may be regarded as covector valued forms. For example, a surface force $\mathbf{t}_{\mathcal{R}}$ may be identified with a section $\hat{\mathbf{t}}_{\mathcal{R}}$ of $\bigwedge^{m-1}\left(T(\partial \mathcal{R}), W^{*}\right)$ by

$$
\hat{\mathbf{t}}_{\mathcal{R}}\left(v_{1}, \ldots, v_{m-1}\right)(w)=\mathbf{t}_{\mathcal{R}}(w)\left(v_{1}, \ldots, v_{m-1}\right) .
$$

so we have an isomorphism of $\bigwedge^{m-1}\left(T \partial \mathcal{R}, W^{*}\right)$ with $L\left(W, \bigwedge^{m-1}\left(T^{*} \partial \mathcal{R}\right)\right)$.

The Cauchy postulates for forces are analogous to those pertaining to the scalar valued properties. The body term, $\mathbf{b}_{\mathcal{R}}$ is assumed to be independent of $\mathcal{R}$ (and is omitted in the spacetime formulation anyhow). The local dependence on the tangent hyperplane is now provided by a section

$$
\Sigma: G_{m-1}(T \mathcal{U}) \rightarrow L\left(\pi_{G}^{*}(W), \bigwedge^{m-1}\left(G_{m-1}(T \mathcal{U})\right)^{*}\right)
$$

where $\pi_{G}^{*}(W)$ is the pull-back of the vector bundle $W$ by the projection of the Grassmann bundle onto $G_{m-1}(T \mathcal{U})$ (see diagram).

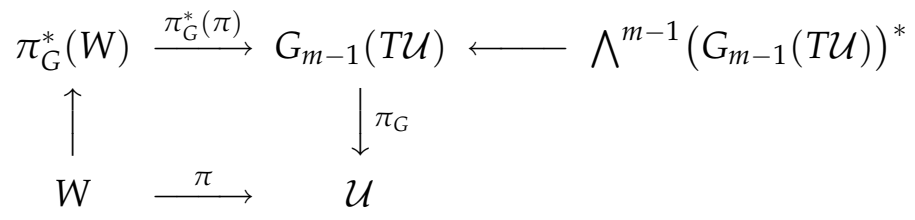

The boundedness postulate, the analog of GC1, requires that there is a section $S$ of the bundle of linear mappings $L\left(J^{1}(W), \bigwedge^{m}\left(T^{*} \mathcal{U}\right)\right)$ such that

$$
\left|F_{\mathcal{R}}(w)\right|=\left|\int_{\mathcal{R}} \mathbf{b}(w)+\int_{\partial \mathcal{R}} \mathbf{t}_{\mathcal{R}}(w)\right| \leq \int_{\mathcal{R}}\left|S\left(j^{1}(w)\right)\right| .
$$


Here, $J^{1}(W)$ is the first jet bundle of $W, j^{1}(w)$ is the first jet of the section $w$, and the absolute value of an $m$-form $\theta, S\left(j^{1}(w)\right)$ in this case, is given as

$$
|\theta(x)|=\left\{\begin{aligned}
\theta(x) & \text { if } \theta(x) \text { is positively oriented, } \\
-\theta(x) & \text { if } \theta(x) \text { is negatively oriented, }
\end{aligned}\right.
$$

relatively to the orientation chosen on $\mathcal{U}$.

The resulting generalized version of Cauchy's theorem states that there is a unique section $\sigma$ of $\bigwedge^{m-1}\left(T \mathcal{U}, W^{*}\right) \cong L\left(W, \bigwedge^{m-1}\left(T^{*} \mathcal{U}\right)\right)$, called the Cauchy stress such that $\mathbf{t}_{\mathcal{R}}(w)=\iota^{*}(\sigma \circ w)$. We will write $\sigma(w)$ for $\sigma \circ w$ and $\iota^{*}(\sigma)$ for $\iota^{*} \circ \sigma$ so we have the Cauchy formula $\mathbf{t}_{\mathcal{R}}=\iota^{*}(\sigma)$ in analogy with the scalar case (with the difference that the forms are vector valued now).

Using Stokes' theorem, the action of $F_{\mathcal{R}}$ may now be rewritten using an integral over $\mathcal{R}$ of $\mathcal{R}$-independent forms and without a boundary term as

$$
F_{\mathcal{R}}(w)=\int_{\mathcal{R}}(d \sigma(w)+\mathbf{b}(w)) .
$$

Assume that $\left(x^{i}, w^{\alpha}\right)$ are local vector bundle coordinates in a neighborhood $\pi^{-1}(U) \subset W, U \subset \mathcal{U}$ with local basis elements $\left\{e_{\alpha}\right\}$ so a section of $W$ is represented locally by $w^{\alpha} e_{\alpha}$. Then, denoting the dual base vectors by $\left\{e^{\alpha}\right\}$ a stress $\sigma$ is represented locally by

$$
\sigma_{\alpha 1 \ldots . . . . . m} e^{\alpha} \otimes d x^{1} \wedge \ldots \wedge \widehat{d x^{k}} \wedge \ldots \wedge d x^{m},
$$

where a "hat" indicates the omission of an item (an index or a factor). The value of $\sigma(w)$ is represented locally by

$$
\sigma_{\alpha 1 \ldots \hat{k} \ldots m} w^{\alpha} d x^{1} \wedge \ldots \wedge \widehat{d x^{k}} \wedge \ldots \wedge d x^{m} .
$$

\section{VARIATIONAL STRESSES}

Let $\pi: W \rightarrow \mathcal{U}$ be a vector bundle as in the previous section. A variational stress density is a section of $L\left(J^{1}(W), \bigwedge^{m}\left(T^{*} \mathcal{U}\right)\right)$.

For the vector bundle coordinates $\left(x^{i}, w^{\alpha}\right), i=1, \ldots, m, \alpha=1, \ldots, \operatorname{dim}\left(W_{x}\right)$, the jet of a section is represented locally by the functions $\left\{w^{\alpha}\left(x^{i}\right), w_{j}^{\beta}\left(x^{k}\right)\right\}$, where a subscript following a comma indicates partial differentiation. A variational stress density will be represented locally by the functions $\left\{S_{\alpha 1 \ldots m}, S_{\beta 1 \ldots}^{j}\right.$ so that the single component of the $m$-form $S\left(j^{1}(w)\right)$ in this coordinate system is

$$
S\left(j^{1}(w)\right)_{1 \ldots m}=S_{\alpha 1 \ldots m} w^{\alpha}+S_{\beta 1 \ldots m}^{j} w_{, j}^{\beta} .
$$

Note that the notation distinguishes between the components of $S$ that are dual to the values of the section and those dual to the derivatives by the number of indices only. The next few paragraphs motivate the introduction of variational stress densities. 
Variational stress theory is formulated usually in a particular frame where the space $(m-1)$-dimensional manifold $\mathcal{M}$ is a global slice of spacetime and $\mathcal{U}$ is interpreted as the $(m-1)$-dimensional material universe manifold. In such a situation, for any body $\mathcal{R}$-an $(m-1)$-dimensional compact submanifold with boundary of $\mathcal{U}$-one may consider configurations of the body in space defined as embeddings of $\mathcal{R}$ in $\mathcal{M}$.

The rational behind the variational formulation of stress theory is the framework for mechanical theories where a configuration manifold is constructed for the system under consideration, generalized velocities are defined as elements of the tangent bundle to the configuration manifold, and generalized forces are defined as elements of the cotangent bundle of the configuration space. For the mechanics of continuous bodies in space, the natural topology for the collection of embeddings is the $C^{1}$ topology for which the collection of embeddings is open in the collection of all $C^{1}$ mappings of the body into space. Using this topology, the tangent space to the configuration manifold at the configuration $\kappa: \mathcal{R} \rightarrow \mathcal{M}$ is $C^{1}\left(\kappa^{*}(T \mathcal{M})\right)$, the Banachable space of $C^{1}$ sections of the pull-back $\kappa^{*}(T \mathcal{M})$. Thus, forces in continuum mechanics are elements of $C^{1}\left(\kappa^{*}(T \mathcal{M})\right)^{*}$-continuous, linear functionals on the space of differentiable vector fields equipped with the $C^{1}$ topology.

The basic representation theorem (see [7]) states that a force functional $F \in C^{1}\left(\kappa^{*}(T \mathcal{M})\right)^{*}$ may be represented by a measure on $\mathcal{U}$-the variational stress measure-valued in $J^{1}\left(\kappa^{*}(T \mathcal{M})\right)^{*}$, the dual of the first jet bundle $J^{1}\left(\kappa^{*}(T \mathcal{M})\right)$ $\mathcal{U}$. The evaluation of a force $F_{\mathcal{R}}$ on the generalized velocity $w$ is

$$
F_{\mathcal{R}}(w)=\int_{\mathcal{R}} d \mu\left(j^{1}(w)\right)
$$

where $\mu$ is the $J^{1}\left(\kappa^{*} T \mathcal{M}\right)^{*}$-valued measure-a section Schwartz distribution.

Assuming that $\mathcal{K}$ is defined on all the material universe $\mathcal{U}$, we use the notation $W$ for $\kappa^{*}(T \mathcal{M})$. This vector bundle can be restricted to the individual bodies, and with some abuse of notation, we use the same notation for both the bundle and its restrictions to the individual bodies.

In the smooth case, a variational stress measure is given in terms of a section $S$ of $L\left(J^{1}(W), \bigwedge^{m-1}\left(T^{*} \mathcal{U}\right)\right.$ ) (recalling the $\mathcal{U}$ is now the material manifold with dimension $m-1)$ so

$$
F_{\mathcal{R}}(w)=\int_{\mathcal{R}} S\left(j^{1}(w)\right) .
$$

Since in the sequel we consider only the smooth case, we will use "variational stresses" to refer to the densities. 
6. The Relation between the Cauchy Approach And the VARIATIONAL APPROACH

In [11] we define a canonical mapping

$$
p_{\sigma}: L\left(J^{1}(W), \bigwedge^{m}\left(T^{*} \mathcal{U}\right)\right) \rightarrow L\left(W, \bigwedge^{m-1}\left(T^{*} \mathcal{U}\right)\right),
$$

that assigns to a variational stress density $S$ a Cauchy stress $\sigma$ satisfying the following relation. At every $x \in \mathcal{U}$ (we suppress the evaluation at $x$ in the notation)

$$
\phi \wedge \sigma(w)=S\left(j_{\phi \otimes w}\right)
$$

for any 1 -form $\phi$. Here, $j_{\phi \otimes w}$ is roughly the jet at $x$ of a section whose value is $0 \in W_{x}$ and its derivative is $\phi \otimes w$. More precisely, if $u: \mathcal{U} \rightarrow W$ is the section whose first jet at $x$ is $j_{\phi \otimes w}$, then, $u$ satisfies the following conditions: $u(x)=0$; denoting the zero section of $W$ by $0, T_{x} u-T_{x} 0 \in L\left(T_{x} \mathcal{U}, T_{0(x)} W_{x}\right)$ induces the linear mapping $\phi \otimes w$ through the isomorphism of $T_{0(x)} W_{x}$ with $W_{x}$. The local representation of $p_{\sigma}$ is as follows. If $\sigma=p_{\sigma}(S)$, then, using the local representatives of $\sigma$ and $S$ as in the previous sections,

$$
\sigma_{\beta 1 \ldots \hat{\ldots} . . . m}=(-1)^{i-1} S_{\beta 1 \ldots m}^{+i}, \quad(\text { no sum over } i) \text {. }
$$

The mapping $p_{\sigma}$ is clearly linear and surjective.

Given a variational stress density $S$ its generalized divergence Div $S$ is the section of $L\left(W, \bigwedge^{m}\left(T^{*} \mathcal{U}\right)\right)$ defined by

$$
\operatorname{Div} S(w)=d\left(p_{\sigma}(S)(w)\right)-S\left(J^{1}(w)\right) .
$$

The local expression for $\operatorname{Div} S(w)$ is

$$
\left(S_{\alpha 1 \ldots m, i}^{i}-S_{\alpha 1 \ldots m}\right) w^{\alpha} d x^{1} \wedge \ldots \wedge d x^{m},
$$

which shows that Div $S$ depends only on the values of $w$ and not its derivative. With these definitions one obtains that

$$
\int_{\mathcal{R}} S\left(j^{1}(w)\right)=\int_{\mathcal{R}} \mathbf{b}_{\mathcal{R}}(w)+\int_{\partial \mathcal{R}} \mathbf{t}_{\mathcal{R}}(w)
$$

where $\mathbf{t}_{\mathcal{R}}(w)=\iota_{\mathcal{R}}^{*}\left(p_{\sigma}(S)(w)\right)$ and $\operatorname{Div} S+\mathbf{b}_{\mathcal{R}}=0$. We conclude that every variational stress induces a unique force system $\left\{\left(\mathbf{b}_{\mathcal{R}}, \mathbf{t}_{\mathcal{R}}\right)\right\}$ through the Cauchy stress it induces and its divergence. Actually, we obtained a decomposition of $S\left(j^{1}(w)\right)$ into an exact differential and a term that is linear in the values of $w$.

The converse is also true. If we have a force system that satisfies Cauchy's postulates, then, the induced Cauchy stress enables us to define a section $S$ of $L\left(J^{1}(W), \bigwedge^{m}\left(T^{*} \mathcal{U}\right)\right)$ by $S\left(j^{1}(w)\right)=\mathbf{b}(w)+d \sigma(w)$. Clearly, writing the local expression for $S$, it is linear in the jet of $w$. Hence,

$$
F_{\mathcal{R}}(w)=\int_{\mathcal{R}} \mathbf{b}(w)+\int_{\mathcal{R}} d \sigma(w)=\int_{\mathcal{R}} S\left(j^{1}(w)\right) .
$$


If for a given variational stress $\mathbf{b}=\operatorname{Div} S=0$, then, $S\left(j^{1}(w)\right)=d p_{\sigma}(S)(w)$.

Thus, we have a complete correspondence between the Cauchy approach and the variational approach to stress theory.

\section{STRESS-ENERGY TENSORS}

Following the interpretation of the flux form $J$ as an object generalizing the velocity vector field, we may consider stress theory on spacetime $\mathcal{U}$ where we set $W=\bigwedge^{m-1}\left(T^{*} \mathcal{U}\right)$. To emphasize this we may write

$$
F_{\mathcal{R}}(J)=\int_{\partial \mathcal{R}} \mathbf{t}_{\mathcal{R}}(J) .
$$

Here, the boundary term $\mathbf{t}_{\mathcal{R}}$ is a section of $L\left(\bigwedge^{m-1}\left(T^{*} \mathcal{U}\right), \bigwedge^{m-1}\left(T^{*} \partial \mathcal{R}\right)\right)$. Note that for the spacetime formulation the term involving $\mathbf{b}_{\mathcal{R}}$ is omitted. Assuming that the generalized Cauchy postulates hold for $\mathbf{t}_{\mathcal{R}}$, the Cauchy stress $\sigma$ is a section of $L\left(\bigwedge^{m-1}\left(T^{*} \mathcal{U}\right), \bigwedge^{m-1}\left(T^{*} \mathcal{U}\right)\right)$. Finally, Div $S=0$ and $d \sigma(J)=S\left(j^{1}(J)\right)$.

The situation may be described generally as follows. We started with an extensive property $p$, given in terms of the flux densities $\tau_{\mathcal{R}}$ for the various control regions $\mathcal{R}$ in spacetime. The source term for property $p$ is $s$ and assuming the Cauchy postulates are satisfied the property $p$ has a flux form $J$. We now consider a second property, the $q$ property, whose flux densities $\tau_{\mathcal{R}}^{(q)}$ for the various control regions and source term $s^{(q)}$ satisfy the conservation equation

$$
\int_{\partial \mathcal{R}} \tau_{\mathcal{R}}^{(q)}=\int_{\mathcal{R}} s^{(q)} .
$$

Again, assuming the Cauchy postulates hold for the property $q$, we have the corresponding flux form $J^{(q)}$ satisfying $\tau_{\mathcal{R}}^{(q)}=\iota^{*}\left(J^{(q)}\right)$ and the conservation equation has the differential representation $d J^{(q)}=s^{(q)}$.

We will say that the property $q$ is a resource for the property $p$ if the flux density $\tau_{\mathcal{R}}^{(q)}$ depends pointwise linearly on the flux form $J$ of the property $p$. Thus, there is a section $\mathbf{t}_{\mathcal{R}}$ as above such that $\tau_{\mathcal{R}}^{(q)}=\mathbf{t}_{\mathcal{R}}(J)$.

In this framework, the Cauchy theorem implies that

$$
\begin{aligned}
\iota^{*}\left(J^{(q)}\right) & =\tau_{\mathcal{R}}^{(q)} \\
& =\mathbf{t}_{\mathcal{R}}(J) \\
& =\iota^{*}(\sigma(J)),
\end{aligned}
$$

for the inclusion $\iota$ of an arbitrary region, so $J^{(q)}=\sigma(J)$. In other words, the Cauchy stress transforms the flux of the property $p$ to the flux of the resource that $p$ uses-the property $q$. The source term for the property $q$ is now given by $s^{(q)}=d \sigma(J)$. 
Naturally, in the sequel we will be concerned primarily with the energy resource.

\section{RePresentations of Force DEnSities AND STRESS-ENERGY TENSORS}

For the situation under consideration a force density (the analog of $\mathbf{b}_{\mathcal{R}}$ if considered) is given in terms of a section of $L\left(\bigwedge^{m-1} W^{*}, \bigwedge^{m} W^{*}\right)$. Such sections have simple representations as follows.

For a vector space $\mathbf{W}$ with dimension $m$, consider, the space of linear mappings $\left(\wedge^{p} \mathbf{W}^{*}\right)^{\top}=L\left(\Lambda^{p} \mathbf{W}^{*}, \bigwedge^{m} \mathbf{W}^{*}\right)$. Define the mapping $\wedge^{p}: \bigwedge^{m-p} \mathbf{W}^{*} \rightarrow$ $\left(\wedge^{p} \mathbf{W}^{*}\right)^{\top}$ by $\wedge^{p}(\alpha)(\beta)=\alpha \wedge \beta$.

Clearly $\wedge^{p}$ is a linear mapping between the two spaces. In addition, as $\bigwedge^{m} \mathbf{W}^{*}$ is 1-dimensional, $\operatorname{dim}\left(\bigwedge^{p} \mathbf{W}^{*}\right)^{\top}=\operatorname{dim}\left(\bigwedge^{p} \mathbf{W}^{*}\right)=\operatorname{dim}\left(\bigwedge^{m-p} \mathbf{W}^{*}\right)$. Thus, $\wedge^{p}$ is an isomorphism if $\operatorname{Kernel}\left(\wedge^{p}\right)=\{0\}$. It is clear however that if $\wedge^{p}(\alpha)(\beta)=\alpha \wedge \beta=0$ for all $\beta$, then $\alpha=0$.

We may conclude for example that a body force density is of the form $A \wedge J$ for a 1 -form $A$.

As the stress-energy tensor is now a section of $L\left(\bigwedge^{m-1}\left(T^{*} \mathcal{U}\right), \bigwedge^{m-1}\left(T^{*} \mathcal{U}\right)\right)$, it is locally represented by a matrix with respect to a local basis of $\bigwedge^{m-1}\left(T^{*} \mathcal{U}\right)$. We will make below some further observations regarding the representations of stresses.

Assume that a volume element $\theta$ is given on $\mathcal{U}$. Then, we may use the vector bundle isomorphism

$$
i_{\theta}: \bigwedge^{m-1}\left(T^{*} \mathcal{U}\right) \rightarrow T \mathcal{U}
$$

to represent the section $\sigma$ of $L\left(\bigwedge^{m-1}\left(T^{*} \mathcal{U}\right), \bigwedge^{m-1}\left(T^{*} \mathcal{U}\right)\right)$ by a section $\tilde{\sigma}$ of $L(T \mathcal{U}, T \mathcal{U})$ satisfying $\tilde{\sigma} \circ i_{\theta}=i_{\theta} \circ \sigma$.

Let us consider the relation between the local representation of $\sigma$ and and the local representation $\tilde{\sigma}_{i}^{j} d x^{i} \otimes \frac{\partial}{\partial x^{j}}$ of $\tilde{\sigma}$. To represent $\sigma$ locally, we will use the notation $\hat{e}^{i}$ for the basis element $d x^{1} \wedge \ldots \wedge \widehat{d x^{i}} \wedge \ldots \wedge d x^{m}$ of $\wedge^{m-1}\left(T^{*} \mathcal{U}\right)$. Thus, the flux form $J$ is represented locally by $\hat{J}_{i} \hat{e}^{i}$, and the stress is represented locally in the form $\hat{\sigma}_{i}^{j} \hat{e}_{j} \otimes \hat{e}^{i}$, where $\left\{\hat{e}_{j}\right\}$ is the dual basis to $\left\{\hat{e}^{i}\right\}$.

If the volume element $\theta$ is represented locally by $r d x^{1} \wedge \ldots \wedge d x^{m}$, the action of $i_{\theta}$ is given locally by

$$
\hat{J}_{i} \hat{e}^{i} \mapsto \sum_{i}(-1)^{i+1} \frac{1}{r} \hat{J}_{i} \frac{\partial}{\partial x^{i}}
$$

(we use the summation symbol as the summation convention cannot be used on the right). Thus, $i_{\theta}(\sigma(J))$ is represented by

$$
\sum_{j}(-1)^{j+1} \frac{1}{r} \hat{\sigma}_{j}^{i} \hat{J}_{i} \frac{\partial}{\partial x^{j}},
$$


and $\tilde{\sigma}\left(i_{\theta}(J)\right)$ is represented by

$$
\sum_{i}(-1)^{i+1} \frac{1}{r} \tilde{\sigma}_{i}^{j} \hat{J}_{i} \frac{\partial}{\partial x^{j}} .
$$

Hence, the relation between $\sigma$ and $\tilde{\sigma}$ is represented locally as

$$
\tilde{\sigma}_{k}^{j}=(-1)^{j+k} \hat{\sigma}_{j}^{k}
$$

It is interesting to note that the volume element does not enter the last relation and one may attempt to arrive at a natural isomorphism between the bundles $L\left(\bigwedge^{m-1}\left(T^{*} \mathcal{U}\right), \bigwedge^{m-1}\left(T^{*} \mathcal{U}\right)\right)$ and $L(T \mathcal{U}, T \mathcal{U})$. Such a natural isomorphism can be constructed as follows. Consider the tensor product $T^{*} \mathcal{U} \otimes \mathcal{U} T \mathcal{U}$. This tensor product is naturally isomorphic to $L(T \mathcal{U}, T \mathcal{U})$. For an element $\tilde{\sigma}=\tilde{\sigma}_{i}^{j} \phi^{i} \otimes v_{j}$ in $T^{*} \mathcal{U} \otimes \mathcal{U} T \mathcal{U}, v_{j} \in T_{x} \mathcal{U}, \phi^{i} \in T_{x}^{*} \mathcal{U}$, set

$$
\sigma: \bigwedge^{m-1}\left(T^{*} \mathcal{U}\right) \rightarrow \bigwedge^{m-1}\left(T^{*} \mathcal{U}\right)
$$

by

$$
\begin{aligned}
\sigma(J) & \left.=\tilde{\sigma}_{i}^{j} v_{j}\right\lrcorner\left(\phi^{i} \wedge J\right) \\
& \left.=\tilde{\sigma}_{i}^{j}\left(\phi^{i}\left(v_{j}\right) J-\phi^{i} \wedge\left(v_{j}\right\lrcorner J\right)\right) .
\end{aligned}
$$

We note that $\sigma$ is indeed linear in $J$. Since $\sigma$ depends linearly on the $\sigma^{i}$ and on the $\phi^{j}$, it depends linearly on the elements of the tensor product.

For the local coordinates $\left\{x^{i}\right\}$, let us determine the stress $\sigma$ induced by the linear mapping $\tilde{\sigma} \in L(T \mathcal{U}, T \mathcal{U})$ represented locally by the tensor $\tilde{\sigma}_{i}^{j} d x^{i} \otimes \frac{\partial}{\partial x^{j}}$. By definition, $\sigma(J)$ is represented by (the sum on $i$ is explicitly written)

$$
\begin{aligned}
\left.\sum_{i} \tilde{\sigma}_{i}^{j} \frac{\partial}{\partial x^{j}}\right\lrcorner\left(d x^{i} \wedge J\right) & \left.=\sum_{i} \tilde{\sigma}_{i}^{j} \frac{\partial}{\partial x^{j}}\right\lrcorner\left(d x^{i} \wedge\left(\hat{J}_{k} d x^{1} \wedge \ldots \wedge \widehat{d x^{k}} \wedge \ldots \wedge d x^{m}\right)\right) \\
& \left.=\sum_{i} \tilde{\sigma}_{i}^{j} \frac{\partial}{\partial x^{j}}\right\lrcorner\left((-1)^{i+1} \hat{J}_{i} d x^{1} \wedge \ldots \wedge d x^{m}\right) \\
& =\sum_{i} \tilde{\sigma}_{i}^{j}(-1)^{i+j} \hat{J}_{i} d x^{1} \wedge \ldots \wedge \widehat{d x^{j}} \wedge \ldots \wedge d x^{m} \\
& =\sum_{i} \tilde{\sigma}_{i}^{j}(-1)^{i+j} \hat{J}_{i} \hat{e}^{j} \\
& =\sum_{i} \hat{\sigma}_{j}^{i} \hat{J}_{i} \hat{e}^{j} .
\end{aligned}
$$


Hence, the matrix representing $\sigma$ is $\hat{\sigma}_{j}^{i}=(-1)^{i+j} \tilde{\sigma}_{i}^{j}$. We conclude that $(*)$ is indeed the natural, invariant representation of the isomorphism between the bundles $L\left(\bigwedge^{m-1}\left(T^{*} \mathcal{U}\right), \bigwedge^{m-1}\left(T^{*} \mathcal{U}\right)\right)$ and $L(T \mathcal{U}, T \mathcal{U})$. This motivates even further the interpretation of the Cauchy stress as a transformation operating on the flux or velocity field of the property $p$ to give the flux form for the energy or velocity of the generalized energy points.

\section{Example: The Maxwell Stress-Energy Tensor Without A METRIC}

As an example for the foregoing analysis, we consider a generalization of the stress-energy tensor of classical electromagnetism to the setting where a metric is not available. We assume that there is a volume element on the 4-dimensional $\mathcal{U}$. The following setting is also independent of any relation between the Maxwell 2-form and the Faraday 2-form such as the relations between the fields $(\mathbf{E}, \mathbf{B})$ and $(\mathbf{D}, \mathbf{H})$ in vacuum. The extensive property under consideration is of course the electric charge and $J$ is the chargecurrent density-a 3-form. The conservation of charge implies that $d J=0$ and the Maxwell 2-form $\mathfrak{g}$ is a flow potential for the flux form so $J=d \mathfrak{g}$. For a 1-form $A$-the vector potential, the energy source density is $A \wedge J$. It follows that the Faraday 2-form $\mathfrak{f}=d A$ satisfies $d \mathfrak{f}=0$.

Thus, assuming that a volume element $\theta$ is given on $\mathcal{U}$, we set $w=i_{\theta}(J)$ and define the stress-energy tensor as the section $\sigma$ of $L\left(\bigwedge^{m-1}\left(T^{*} \mathcal{U}\right), \wedge^{m-1}\left(T^{*} \mathcal{U}\right)\right)$ by (c.f. [12] p. 36 for the closest expression we found)

$$
\left.\left.\sigma(J)=\left(i_{\theta}(J)\right\lrcorner \mathfrak{g}\right) \wedge \mathfrak{f}-\left(i_{\theta}(J)\right\lrcorner \mathfrak{f}\right) \wedge \mathfrak{g} .
$$

Alternatively, using

$$
w\lrcorner(\mathfrak{g} \wedge \mathfrak{f})=(w\lrcorner \mathfrak{g}) \wedge \mathfrak{f}+\mathfrak{g} \wedge(w\lrcorner \mathfrak{f}),
$$

the definition of the electromagnetic stress-energy tensor may also be written as

$$
\left.\left.\sigma(J)=i_{\theta}(J)\right\lrcorner(\mathfrak{g} \wedge \mathfrak{f})-2\left(i_{\theta}(J)\right\lrcorner \mathfrak{f}\right) \wedge \mathfrak{g} .
$$

Note that the matrix of the Cauchy stress with respect to the natural basis of the space of $(m-1)$-forms is related to the usual matrix of the stressenergy-momentum tensor as discussed in the previous section.

We now consider the energy source term $d \sigma(J)$. Using $w=i_{\theta}(J)$ one obtains

$$
\begin{aligned}
d \sigma(J) & \left.\left.=d\left(\left(i_{\theta}(J)\right\lrcorner \mathfrak{g}\right) \wedge \mathfrak{f}-\left(i_{\theta}(J)\right\lrcorner \mathfrak{f}\right) \wedge \mathfrak{g}\right) \\
& =d(w\lrcorner \mathfrak{g}) \wedge \mathfrak{f}-(w\lrcorner \mathfrak{g}) \wedge d \mathfrak{f}+(w\lrcorner \mathfrak{f}) \wedge d \mathfrak{g}-d(w\lrcorner \mathfrak{f}) \wedge \mathfrak{g} \\
& =d(w\lrcorner \mathfrak{g}) \wedge \mathfrak{f}+(w\lrcorner \mathfrak{f}) \wedge J-d(w\lrcorner \mathfrak{f}) \wedge \mathfrak{g},
\end{aligned}
$$

where Maxwell's equations were used to arrive at the last line. Using the identity $\left.d(w\lrcorner \alpha)=\mathcal{L}_{w} \alpha-w\right\lrcorner d \alpha$, for any differential form $\alpha$, we have

$$
\left.\left.d \sigma(J)=(w\lrcorner \mathfrak{f}) \wedge J+\left(\mathcal{L}_{w} \mathfrak{g}-u\right\lrcorner d \mathfrak{g}\right) \wedge \mathfrak{f}-\left(\mathcal{L}_{w} \mathfrak{f}-w\right\lrcorner d \mathfrak{f}\right) \wedge \mathfrak{g} .
$$


Finally, as $w\lrcorner J=0$, Maxwell's equations give

$$
d \sigma(J)=(w\lrcorner \mathfrak{f}) \wedge J+\left(\mathcal{L}_{w} \mathfrak{g}\right) \wedge \mathfrak{f}-\left(\mathcal{L}_{w} \mathfrak{f}\right) \wedge \mathfrak{g} .
$$

It is noted that the term $(w\lrcorner \mathfrak{f}) \wedge J$ represents the power of the Lorentz force. In addition, in the classical formulation where a metric is available and $\mathfrak{g}=* \mathfrak{f}\left(*\right.$ denotes the Hodge operator), the terms $\left(\mathcal{L}_{w} \mathfrak{g}\right) \wedge \mathfrak{f}$ and $\left(\mathcal{L}_{w} \mathfrak{f}\right) \wedge$ $\mathfrak{g}$ are equal and the energy source density contains the power of the Lorentz force (and energy conservation) only. For an analogous expression where the constitutive relation between $\mathfrak{g}$ and $\mathfrak{f}$ is not specified but a metric is used, see [2, p. 91].

\section{Appendix A. Local Representation of the MAXWELL STRESS-ENERGY TENSOR}

We write the local representation $\hat{\mathfrak{f}}_{i j} d x^{i} \wedge d x^{j}$ of the Faraday 2-form $\mathfrak{f}$ in the form

$$
\left\{\hat{\mathfrak{f}}_{i j}\right\}=\left(\begin{array}{cccc}
0 & -E_{1} & -E_{2} & -E_{3} \\
E_{1} & 0 & B_{3} & -B_{2} \\
E_{2} & -B_{3} & 0 & B_{1} \\
E_{3} & B_{2} & -B_{1} & 0
\end{array}\right),
$$

and the corresponding representation $\hat{\mathfrak{g}}_{i j} d x^{i} \wedge d x^{j}$ for the Maxwell 2-form as

$$
\left\{\hat{\mathfrak{g}}_{i j}\right\}=\left(\begin{array}{cccc}
0 & H_{1} & H_{2} & H_{3} \\
-H_{1} & 0 & D_{3} & -D_{2} \\
-H_{2} & -D_{3} & 0 & D_{1} \\
-H_{3} & D_{2} & -D_{1} & 0
\end{array}\right),
$$

For simplicity of the notation we assume that locally the volume element $\theta$ is of the form $d x^{1} \wedge \cdots \wedge d x^{4}$. Then, $w=i_{\theta}(J)$ is represented by $w^{i}=$ $(-1)^{i+1} \hat{J}_{i}$. With this notation, the matrix $\left\{\hat{\sigma}_{i}^{j}\right\}$ representing the stress-energy tensor is

$$
\left\{\hat{\sigma}_{i}^{j}\right\}=\left(\begin{array}{llll}
\left\{\hat{\sigma}_{i}^{1}\right\} & \left\{\hat{\sigma}_{i}^{2}\right\} & \left\{\hat{\sigma}_{i}^{3}\right\} & \left\{\hat{\sigma}_{i}^{4}\right\}
\end{array}\right),
$$

where,

$$
\begin{array}{r}
\hat{\sigma}_{i}^{1}=\left\{\begin{array}{c}
H_{1} B_{1}+H_{2} B_{2}+H_{3} B_{3}+D_{1} E_{1}+D_{2} E_{2}+E_{3} D_{3} \\
2\left(E_{3} H_{2}-E_{2} H_{3}\right) \\
2\left(E_{3} H_{1}-E_{1} H_{3}\right) \\
2\left(E_{2} H_{1}-E_{1} H_{2}\right)
\end{array}\right\}, \\
2\left(B_{3} D_{2}-B_{2} D_{3}\right) \\
\hat{\sigma}_{i}^{2}=\left\{\begin{array}{c}
H_{1} B_{1}-H_{2} B_{2}-H_{3} B_{3}+E_{1} D_{1}-E_{2} D_{2}-E_{3} D_{3} \\
2\left(E_{1} D_{2}-B_{2} H_{1}\right) \\
2\left(E_{1} D_{3}+B_{3} H_{1}\right)
\end{array}\right\},
\end{array}
$$




$$
\begin{gathered}
\hat{\sigma}_{i}^{3}=\left\{\begin{array}{c}
2\left(B_{3} D_{1}-B_{1} D_{3}\right) \\
2\left(B_{1} H_{2}-E_{2} D_{1}\right) \\
-H_{1} B_{1}+H_{2} B_{2}-H_{3} B_{3}-E_{1} D_{1}+E_{2} D_{2}-E_{3} D_{3} \\
2\left(-E_{2} D_{3}-B_{3} H_{2}\right)
\end{array}\right\}, \\
2\left(B_{2} D_{1}-B_{1} D_{2}\right) \\
2\left(B_{1} H_{3}-E_{3} D_{1}\right) \\
2\left(-E_{3} D_{2}-B_{2} H_{3}\right) \\
\hat{\sigma}_{i}^{4}=\left\{\begin{array}{c}
\left\{H_{3} B_{3}-E_{1} D_{1}-E_{2} D_{2}+E_{3} D_{3}\right.
\end{array}\right\} .
\end{gathered}
$$

Acknowledgements. The author would like to thank Prof. R. Tucker for the useful discussions.

\section{REFERENCES}

[1] L.D. Landau \& E.M. Lifshitz, The Classical Field Theories, Course of Theoretical Physics, Vol. 2, Butterworth, Oxford, 1975.

[2] A. Lichnerowicz, Relativistic Hydrodynamics and Magnetohydrodynamics, Benjamin, New York, 1967,

[3] C.W. Misner, K.S. Thorne \& J.A. Wheeler, Gravitaion, Freeman, San Francisco, 1973.

[4] R.K. Sachs \& H. Wu, General Relativity for Mathematicians, Springer, New-York, 1977.

[5] R.M. Wald, General Relativity, University of Chicago Press, Chicago, 1984.

[6] R. Segev, The geometry of Cauchy's fluxes, Archive for Rational Mechanics and Analysis, 154 (2000) 183-198.

[7] R. Segev, Forces and the existence of stresses in invariant continuum mechanics, Journal of Mathematical Physics 27 (1986) 163-170.

[8] R. Segev \& G. Rodnay, Cauchy's theorem on manifolds, Journal of Elasticity, 56 (2000), $129-144$.

[9] M.J. Gotay \& J.E. Marsden, Stress-energy-momentum tensors and the BelifanteRosenfeld formula, AMS Contemp. Math., 132 (1992), 367-392.

[10] R. Abraham, J.E. Marsden, \& R. Ratiu, Manifolds, Tensor Analysis, and Applications, Springer, New York, 1988.

[11] R. Segev \& G. Rodnay, Divergences of stresses and the principle of virtual work on manifolds, Technische Mechanik, 20 (2000), 129-136.

[12] W. Thirring, A Course in Mathematical Physics 2, Classical Field Theory, Springer-Verlag, New York, 1979.

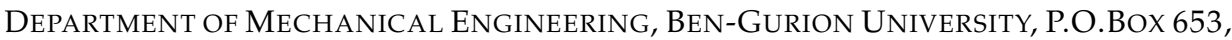
BEER-SHEVA 84105 ISRAEL

E-mail address: rsegev@bgumail.bgu.ac.il 